\title{
Optimized Strategies for Coordinated Spectrum Sensing in Cognitive Radio Networks
}

\author{
Zaili Wang ${ }^{\dagger}$, Zhiyong Feng ${ }^{\ddagger}$, Di Zhang ${ }^{\S}$, Jiantao Xue ${ }^{\llbracket}$, Ping Zhang ${ }^{\$}$ \\ Wireless Technology Innovation Institute \\ Key Laboratory of Universal Wireless Communications, Ministry of Education \\ Beijing University of Posts and Telecommunications, Beijing, 100876, P.R.China \\ Email: $\left\{\right.$ zaili.wang ${ }^{\dagger}$, zhangdiwti $^{\S}$, xuejiantao $\}$ @ gmail.com, $\left\{\right.$ fengzy $^{\ddagger}$, pzhang $\left.{ }^{\$}\right\} @$ bupt.edu.cn
}

\begin{abstract}
In Cognitive Radio Networks, coordinated spectrum sensing is a novel technique that the cognitive terminals are assigned to sense different channels in the same sensing slots in order to obtain the occupancy status of all the channels in every sensing period. In this paper the coordinated spectrum sensing strategies are discussed, the functional architecture is put forward, a Kuhn-Munkres based, alongside with a greedy based algorithm for coordinated sensing strategies arrangement is proposed. Simulation results show that the algorithms can significantly improve the overall sensing performance compared with a basic greedy based algorithm.
\end{abstract}

\section{INTRODUCTION}

Cognitive Radio (CR) has been developing rapidly for the last ten years since it was proposed by Mitola in 1999 [1]. As solutions to the spectrum scarcity problem, CR technologies enable the unauthorized users (Secondary Users, SUs) to utilize the spectrum when it is not in use by the authorized users (Primary Users, PUs). Therefore Cognitive Radio Networks (CRNs) have to be equipped with spectrum sensing functionalities so that they can detect whether the channel is occupied by PU signals. Spectrum sensing has been extensively studied as an indispensable technique for CRNs. A number of sensing algorithms have been put forward, such as energy detection, matched filter detection and cyclostationary feature detection [2]. Furthermore, cooperative spectrum sensing that a controlling node fuses the local sensing results from the cognitive terminals to get a final decision, has also been extensively studied as an approach to improving the sensing performance and overcoming the hidden node problem. Most of the study on cooperative sensing focus on sensing nodes selection [3], data fusion [4], sensing-throughput tradeoff [5] and relevant MAC protocol design [6].

In practice, CRNs usually have numerous potentially utilizable channels for dynamic access. The networks need to get the accessibility of all the channels in each sensing cycle. However, the time length of the sensing slots has to be very short to avoid the harmfully interruption of the communications in CRNs, therefore it is hardly possible for a cognitive terminal (CT) to perform wideband spectrum sensing in a sensing slot due to hardware (e.g. A/D sampling rate) and energy constraints. To solve the problem, an effective way is to assign the terminals to sense different channels in the same sensing slots.
We name the scheme as coordinated spectrum sensing to distinguish it from the conventional cooperative spectrum sensing schemes. In coordinated spectrum sensing, each terminal is assigned to detect a channel and every channel needs to be detected unless the terminals are less than the channels. Moreover, a joint work of coordinated sensing and cooperative sensing is available in case the terminals outnumber the channels, as the terminals assigned to sense the same channel can cooperate to achieve better sensing performance. The idea of coordinated spectrum sensing was proposed in [7] with the name of Frequency-Division Cooperative Spectrum Sensing (FD-CSS) and adopted in the works in [8].

Here comes the vital question in coordinated sensing: how to assign the terminals to sense the channels to achieve the best overall sensing performance? In [8] the terminals are randomly assigned, which may lead to poor system sensing performance. [7] pointed out that the band assignment strategy should be obtained based on the various conditions of the channels at each terminal. However, it is very hard for CRNs to estimate the channel condition of the terminals to the PU transmitters in real-time. In addition, the band assignment algorithm was not discussed in detail in [7].

In this paper we discuss several key issues in coordinated spectrum sensing. First, we put forward a functional architecture in the cognitive base station (CBS) that supports the coordinated sensing procedure. Second, we propose a band assignment strategy for coordinated sensing based on the Kuhn-Munkres Algorithm. Third, we propose a strategy with relatively inferior performance but lower complexity based on the greedy algorithm .

The rest of this paper is organized as follows: Section II illustrates the functional architecture as well as an overview of coordinated sensing procedure. As an important preceding step in coordinated sensing strategy arrangement, the sensing performance evaluation process is discussed in Section III. In Section IV the algorithms for coordinated sensing strategy arrangement are described in detail. The simulation results are shown and discussed in Section $\mathrm{V}$ and the paper is concluded in Section VI. 


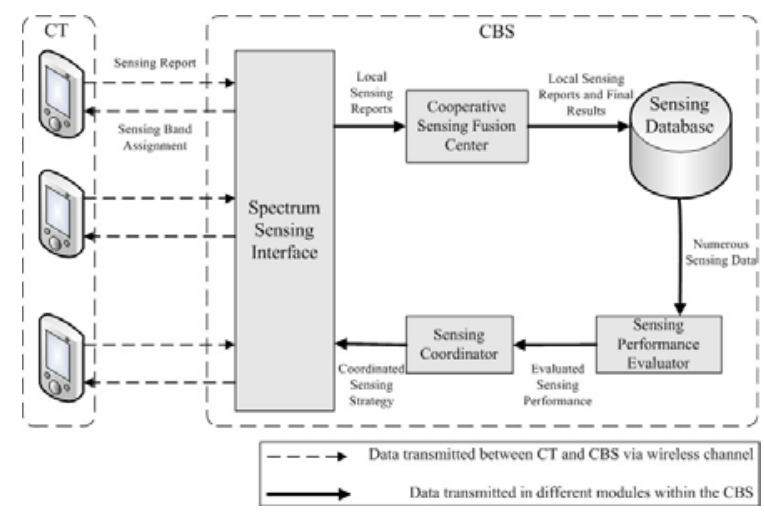

Fig. 1. The functional architecture of coordinated spectrum sensing

\section{An Overview of Coordinated Spectrum Sensing}

\section{A. The Functional Architecture}

This paper is on the ground of a CRN consisted of a CBS and $N$ cognitive terminals under the coverage of it. The spectrum is divided into $M$ channels, marked as $f_{1}, f_{2}, \ldots, f_{M}$. In the $\mathrm{CRN}$, the terminals detect the channels periodically in the sensing slots, reporting the binary results, where 1 indicating PU signals detected and 0 indicating PU signals not detected, to the CBS.

It is obvious that some functional modules have to be appended in the CBS to implement the optimized coordinated sensing strategies. In general, the functional modules should include the followings, as illustrated in Fig. 1:

Fusion Center: Fuses the local sensing results to get final PU occupancy decisions for the respective channels;

Sensing Database: Stores the historical fusion decisions as well as the raw sensing reports from the terminals;

Sensing Performance Evaluator: Extracts relevant data from the Sensing Database and evaluates the sensing performance of the terminals for the channels based on the data;

Sensing Coordinator: Arrange a coordinated sensing strategy on the basis of the evaluated sensing performance.

\section{B. The Coordinated Spectrum Sensing Procedure}

At the terminals, the probability of misdetection $\left(P_{m d}\right)$ and false alarm $\left(P_{f a}\right)$ are two kinds of sensing errors indicating the sensing performance. In this paper energy detection is deployed for local sensing, so according to [9], $P_{f a}$ is expressed as

$$
P_{f a}=\frac{\Gamma\left(u, \frac{\lambda}{2}\right)}{\Gamma(u)}
$$

where $u$ is the time-bandwidth product, $\lambda$ is the detection threshold, $\Gamma($.$) is the gamma function and \Gamma(.,$.$) is the$ incomplete gamma function. The probability of misdetection $\left(P_{m d}\right)$ under Additive White Gaussian Noise (AWGN) channel is given by

$$
P_{m d}=1-Q_{u}(\sqrt{2 \gamma}, \sqrt{\lambda})
$$

where $\gamma$ is the SNR at the CT and $Q_{u}(.,$.$) is the generalized$ Marcum Q-function.
The reported binary local sensing results are fused by the Fusion Center in the CBS. On the criterion of OR-Rule, the probability of misdetection and false alarm of channel $f_{m}$ are

$$
\begin{gathered}
Q_{m d}^{m}=\prod_{f(n)=f_{m}} P_{m d}^{n, m} \\
Q_{f a}^{m}=1-\prod_{f(n)=f_{m}}\left(1-P_{f a}^{n, m}\right)
\end{gathered}
$$

where $f(n)=f_{m}$ means terminal $n$ is assigned to sense $f_{m}$, $P_{m d}^{n, m}$ and $P_{f a}^{n, m}$ are the probability of misdetection and false alarm of terminal $n$ for channel $f_{m}$.

The fused decisions about the occupancy status of the channels, along with the raw reported data from the terminals, are stored in Sensing Database. The Database is updated with the latest reports and decisions, while the expired data are discarded. The data are used to evaluate the sensing performance of the terminals, and a coordinated sensing strategy is derived from the sensing performance by Sensing Coordinator. In the following sections, we focus on the algorithms for optimizing the coordinated spectrum sensing strategies.

\section{The Evaluation of the Sensing Performance}

The evaluation process is an important preceding step, providing the sensing performance information for the coordinated sensing arrangement algorithms. A terminal which just accessed in the network cannot join the coordinated sensing procedure until its sensing performance for the channels is evaluated.

As mentioned above, there are two performance indicators for local spectrum sensing, which are $P_{f a}$ and $P_{m d}$. To have a straight and fair comparison of the sensing performance, $P_{f a}$ is set to be a fixed, unified parameter so that $P_{m d}$ itself can indicate how reliable the sensing results are. However, in practice $P_{m d}$ is not available because in (2), $\gamma$ is not known by the CT. Hence, $P_{m d}$ can just be evaluated in a statistical way.

When accessed to the CRN, a terminal should at first sense all the channels in a round robin schedule in successive sensing slots for $T$ loops (i.e. $M \times T$ slots), reporting the binary sensing results that take part in data fusion. Sensing Performance Evaluator applies a statistical method estimating $P_{m d}$ for sensing the channels [10]. The local sensing reports are stored in matrix

$$
\mathbf{y}^{l o c}=\left[y_{m, t}^{l o c}\right]_{M \times T}
$$

while the fusion decisions are stored as

$$
\mathbf{y}^{f u s}=\left[y_{m, t}^{f u s}\right]_{M \times T}
$$

where $y_{m, t}^{l o c} \in\{0,1\}$ is the $t$ th local sensing report for channel $m$, and $y_{m, t}^{f u s} \in\{0,1\}$ indicates the corresponding fusion decisions. The evaluated $P_{m d}$ of the terminal for channel $m$ is

$$
P_{m d}^{m}=\frac{\sum_{t=1}^{T} y_{m, t}^{l o c}}{\sum_{t=1}^{T} y_{m, t}^{f u s}}
$$


For any terminal, the sensing performance usually varies from different channels because the channels may be used by various PU transmitters that the PU signals have different transmitting power as well as different path loss and shadowing to the sensing terminals. Hence, coordinated spectrum sensing strategies should be carefully designed to achieve optimized overall sensing performance.

\section{Algorithms For Coordinated Spectrum SENSING STRATEgIES}

\section{A. Problem Formulation}

The terminals who have their sensing performance evaluated can join the coordinated spectrum sensing arranged by Sensing Coordinator. Assume that there are $N$ participating terminals for coordinated sensing, and $N>M$ so that several terminals can detect the same channel with cooperative sensing to further improve sensing performance. With $P_{f a}$ fixed and unified, the sensing performance matrix can be defined as

$$
\mathbf{P}_{\mathbf{m d}}=\left[P_{m d}^{n, m}\right]_{N \times M}
$$

where $n=1,2, \cdots, N ; m=1,2, \cdots, M$.

On the criterion of OR-rule in the fusion of the cooperative sensing reports, it is obvious that the probability of misdetection $Q_{m d}^{m}$ will decrease as more terminals join the cooperation for sensing channel $\mathrm{m}$. We define the cooperation gain for terminal $n$ joining the cooperative sensing of channel $m$ as the decrement of misdetection probability

$$
\Delta Q_{m d}^{n, m}=\tilde{Q}_{m d}^{m}-P_{m d}^{n, m} \tilde{Q}_{m d}^{m}
$$

where $\tilde{Q}_{m d}^{m}$ is the misdetection probability for channel $m$ before the joining of terminal $n$.

However, the number of the terminals cooperatively sensing a channel cannot be unlimitedly increased because the probability of false alarm $Q_{f a}$ is simultaneously increasing. The system should set $Q_{f a}$ upper bound for detection as a system requirement. When unified $P_{f a}$ and $Q_{f a}$ values are set for sensing, the maximum number of the terminals cooperatively sensing one channel with OR-rule in data fusion is

$$
N_{\max }^{c o o p}=\left\lfloor\log _{\left(1-P_{f a}\right)}\left(1-Q_{f a}\right)\right\rfloor .
$$

The objective of coordinated spectrum sensing is to assign every terminal with a most appropriate channel to detect, optimizing the overall sensing performance. Each terminal can sense one channel while each channel can be cooperatively sensed by at most $N_{\max }^{c o o p}$ terminals. The overall sensing performance can be indicated by the overall probability of misdetection of the channels, which should be minimized in the coordinated sensing strategy. Furthermore, the assignment matrix $\mathbf{X}=\left[x^{n m}\right]_{N \times M}$ is introduced

$$
x^{n m}= \begin{cases}1 & f(n)=f_{m} \\ 0 & \text { else. }\end{cases}
$$

So the sensing task assignment problem for coordinated sensing can be formulated as

$$
\min _{\mathbf{X}} \quad \sum_{m=1}^{M} Q_{m d}^{m}=\sum_{m=1}^{M}\left(\prod_{x^{n m}=1} P_{m d}^{n, m}\right) ;
$$

$$
\begin{array}{ll}
\text { s.t. } \quad & \sum_{m=1}^{M} x^{n m}=1, \quad n=1,2, \cdots, N ; \\
& 1 \leq \sum_{n=1}^{N} x^{n m} \leq N_{\max }^{\text {coop }}, \quad m=1,2, \cdots, M ; \\
x^{n m} \in\{0,1\}, \quad n=1, \cdots, N ; m=1, \cdots, M .
\end{array}
$$

\section{B. The Kuhn-Munkres Based Algorithm}

Kuhn-Munkres Algorithm [11] [12] is a well-known solution for Linear Assignment Problem (LAP) optimization [13] that works with a pair consisting of an infeasible primal and a feasible dual solution which fulfill the complementarity slackness conditions. The algorithm updates the solutions iteratively until the primal solution becomes feasible, while keeping the complementary slackness conditions fulfilled. At this point the primal solution is also optimal, according to duality theory.

As the sensing task assignment problem is similar to a LAP to some extent, the proposed assignment algorithm for coordinated sensing is designed based on Kuhn-Munkres Algorithm.

In the first step, the algorithm assigns $M$ terminals, one terminal to sense each channel with Kuhn-Munkres Algorithm so that overall $P_{m d}$ is minimized. In each of the subsequent steps, the algorithm assigns one more terminal to cooperatively sense each channel, with maximized total cooperation gain. So the algorithm is realized during an iterative process, in each iteration the problem is a LAP, solved by Kuhn-Munkres Algorithm. At the end of each iteration, $\Delta Q_{m d}$ for the channels are updated, the assigned terminals are excluded from subsequent steps. The algorithm terminates when there are no more unassigned terminals, or every channel has been cooperatively sensed by $N_{\max }^{c o o p}$ terminals. A detailed expression of the algorithm is given as Algorithm 1.

It is obvious that the iteration time of the proposed KuhnMunkres based algorithm is

$$
T_{i t}=\min \left(\lfloor N / M\rfloor, N_{\max }^{c o o p}\right)
$$

As Kuhn-Munkres Algorithm can achieve a worst case time complexity of $\mathrm{O}\left(N^{3}\right)$, the worst case time complexity of the proposed algorithm is $\mathrm{O}\left(N^{3} \cdot T_{i t}\right)$.

\section{The Greedy Based Algorithm}

Although the Kuhn-Munkres based algorithm can achieve polynomial complexity, the complexity is still relatively high, which may hinder its feasibility in practical CRNs. In the followings a greedy based algorithm is proposed, which may not achieve as good performance as the Kuhn-Munkres based one, but have lower complexity and more applicable in practical systems.

The greedy based algorithm is based on the idea that $Q_{m d}^{m}$ can be seen as 1 when there are no terminals assigned to sense channel $m$, so the cooperation gain is $1-P_{m d}^{n, m}$ if $n$ is the first terminal assigned to sense channel $m$. Each time the algorithm finds the maximum cooperation gain value $\Delta Q_{m d}^{*}=$ 


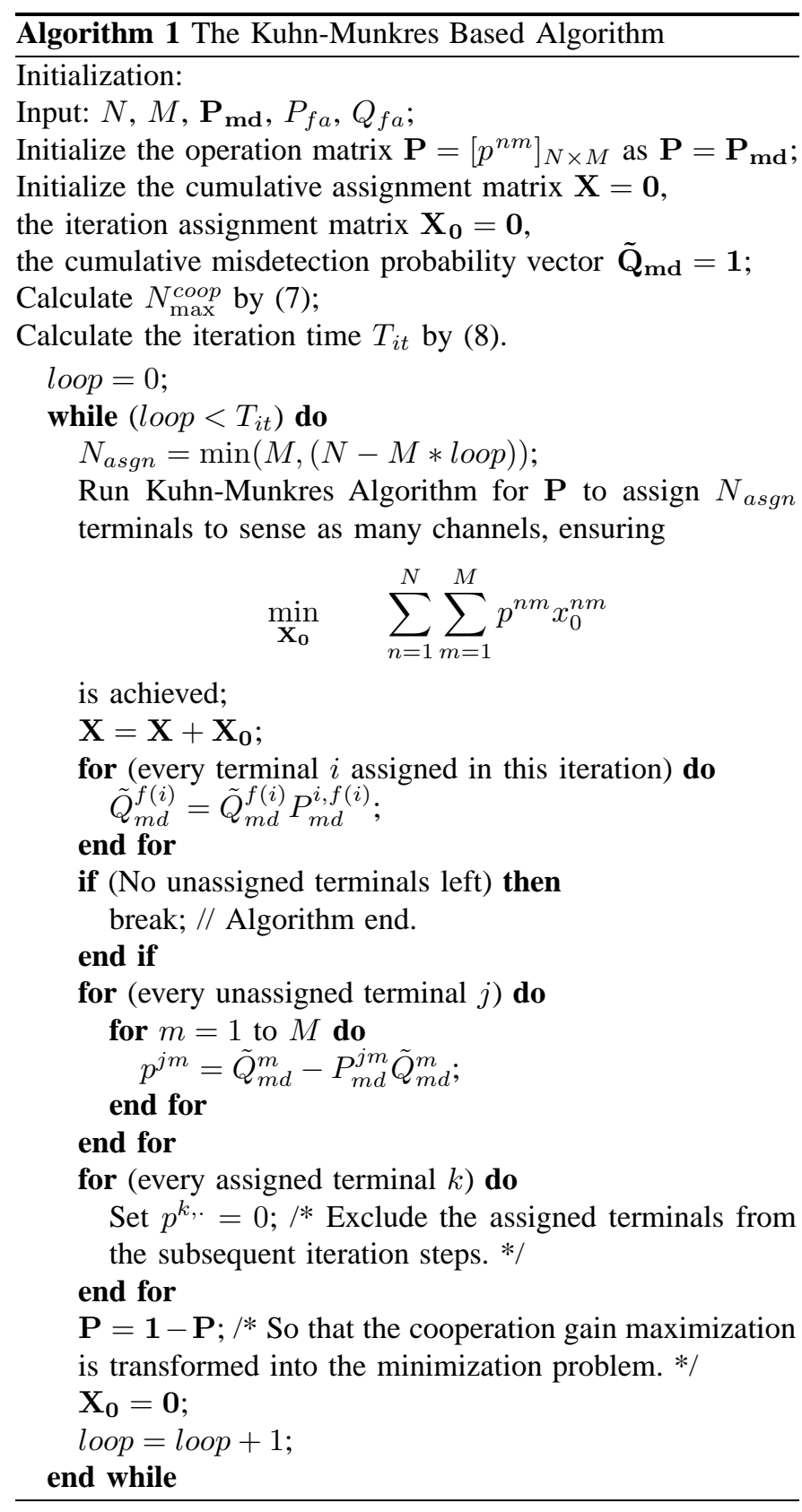

$\Delta Q_{m d}^{n, m}$, then assigns terminal $n$ to sense channel $m$, updates other terminals' cooperation gain for channel $m$, and excludes terminal $n$ from the subsequent steps. Algorithm 2 describes the algorithm in detail.

The algorithm proposed in this subsection can be seen as a sorting procedure followed by the reciprocation of value adjustment and corresponding repositioning in the sorted array. Taking advantage of the data structure of heap, the worst case time complexity of the sorting procedure can achieve $\mathrm{O}(M N$. $\log M N)$, which is around $\mathrm{O}(N \log N)$ when $N$ is large, and the complexity of the following steps is no higher than $\mathrm{O}(N$. $\min \left(N, N_{\max }^{c o o p} * M\right)$ ), which is around $\mathrm{O}(N)$ when $N$ is large. Hence, the time complexity of the greedy based algorithm is around $\mathrm{O}(N \log N)$.

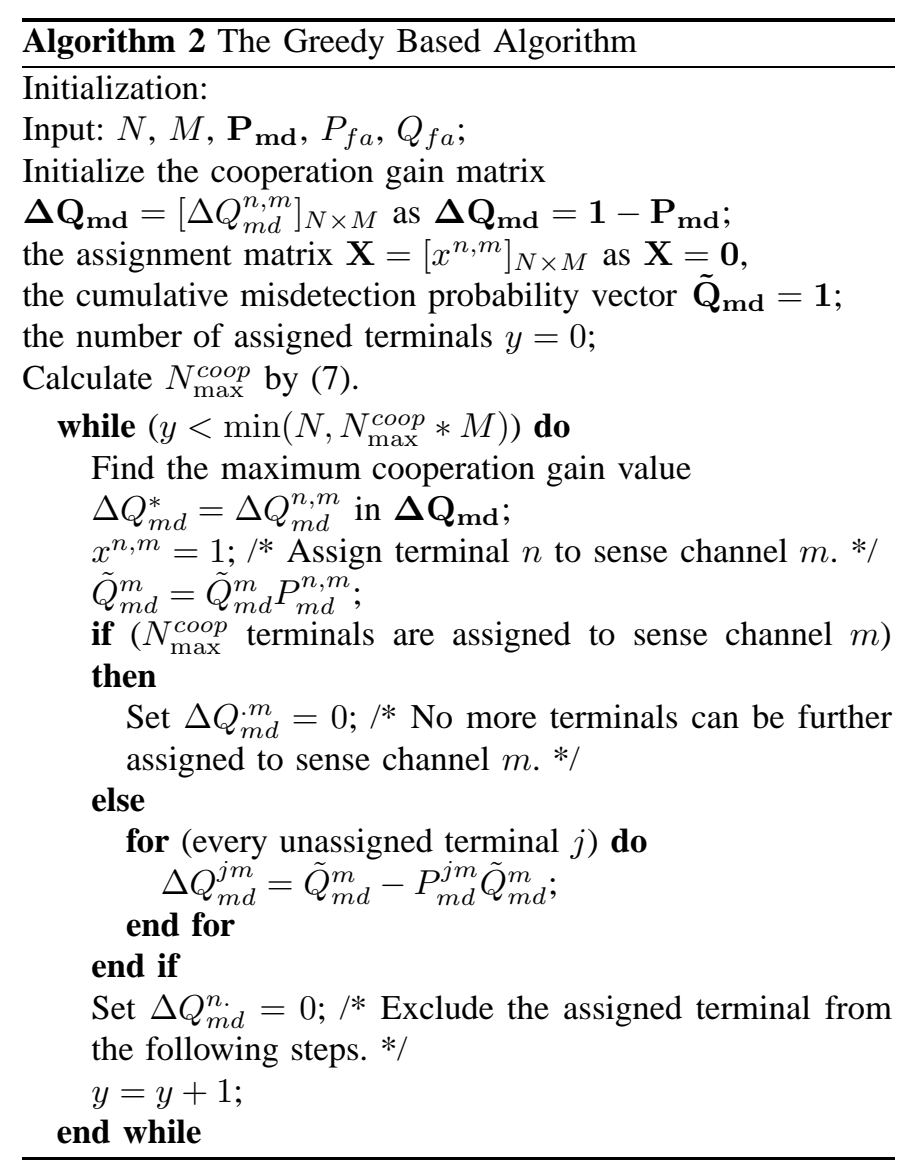

It should be mentioned that when $N \leq M$, cooperative sensing is not available, but the optimized coordinated sensing strategies can still be obtained by the proposed algorithms.

\section{Simulation Results}

In this section we offer the simulation results to demonstrate the performance of the strategies as results of the proposed algorithms in comparison with a basic assignment strategy (hereinafter referred to as Algorithm 3), where each terminal detects the channel which it can sense with the best sensing performance.

In the simulated scenarios, there are 8 channels to be sensed by the CRN. For the fusion of the local sensing reports on the channels, OR-rule is adopted, hence the false alarm probability $P_{f a}$ should be very low to maintain an acceptable $Q_{f a}$. In the simulation $P_{f a}$ is set as 0.01 for all the local sensing and the false alarm probability upper bound $Q_{f a}=0.05$ for cooperative sensing of the channels. Therefore $N_{\max }^{c o o p}$ is 5 according to (7).

The simulation results shown in Fig. 2 demonstrate the average $Q_{m d}$ over different number of terminals with average local $P_{m d}$ around 0.6 and 0.4 respectively. Although $\mathbf{P}_{\text {md }}$ for the scenarios are not listed in detail, the fairness of the comparison is guaranteed as the algorithms are separately executed for the same scenarios. The results show that Algorithm 1 has the best performance, followed closely by Algorithm 2. By contrast, Algorithm 3 has a remarkably poorer performance. 


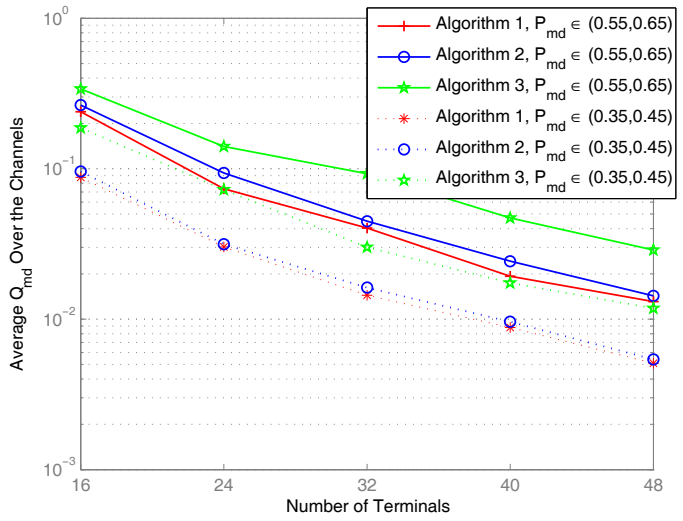

Fig. 2. Average $Q_{m d}$ over different number of terminals, $M=8$

The evaluation of the local sensing performance introduced in Section III is vital for coordinated sensing, but in reality it is hardly possible to evaluate the performance with perfect accuracy. Hence the performance of coordinated sensing strategies with imperfect local sensing performance evaluation should be discussed.

To simplify the problem, we introduce a estimation error factor matrix $\mathbf{e}=e^{n, m}$, where the elements are i.i.d variables following normal distribution: $e^{n, m} \sim N\left(1, \sigma^{2}\right)$. The evaluated local sensing performance with estimation error is

$$
P_{m d, e}^{n, m}=P_{m d}^{n, m} e^{n, m} \quad n=1, \cdots, N ; m=1, \cdots, M .
$$

The results are shown in Fig. 3, indicating that the algorithms are merely affected by estimation error. Moreover, the curve of Algorithm 2 is almost overlapped with that of Algorithm 1 as estimation error gets larger. This means Algorithm 2, with lower complexity, is the most practical algorithm for CRNs when the number of terminals is large. Although Algorithm 1 is less applicable due to its higher complexity, it is also significant because the algorithm leads to an optimal strategy with accurate sensing performance evaluation, providing a lower bound for the average $Q_{m d}$ over the channels.

\section{CONCLUSION}

In this paper we discuss the band-assignment problem for coordinated spectrum sensing in detail. In addition to the functional architecture, two algorithms for the coordinated sensing strategies with different complexity are proposed. From the numerical results, it can be concluded that the greedy based algorithm is the most adoptable in practical CRNs owing to its low complexity, near-optimal performance and robustness to the inaccurate local sensing performance evaluations.

\section{ACKNOWLEDGMENT}

This work is supported in part by National High Technology Research and Development Program of China with NO.2009AA011802, National Basic Research Program of China with NO.2009CB320407, National Key Technology

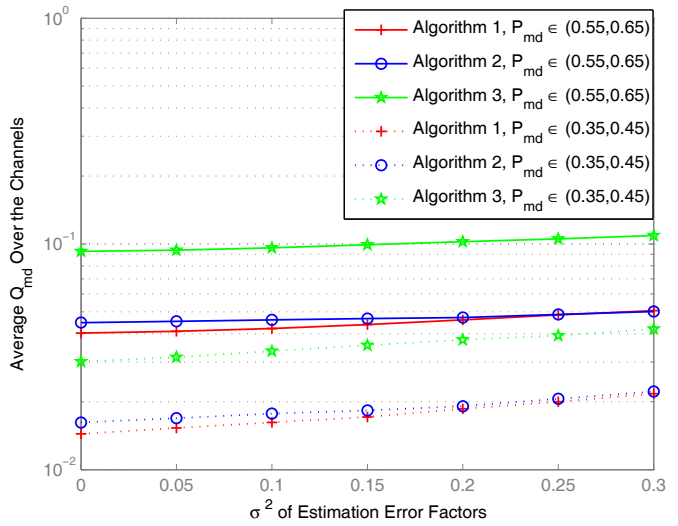

Fig. 3. Average $Q_{m d}$ over different error factors with $M=8, N=32$

R\&D Program of China with NO.2009ZX03007-004 and Key Project of National Natural Science Foundation of China with NO.60832009.

\section{REFERENCES}

[1] J. Mitola and G. Maguire Jr. , "Cognitive Radio: Making Software Radios More Personal", IEEE Personal Communications, vol. 6, no. 4, pp. 1318, Aug. 1999

[2] T. Yucek and H. Arslan, "A Survey of Spectrum Sensing Algorithms for Cognitive Radio Applications", IEEE Communications Surveys \& tutorials, vol. 11, no. 1, pp. 116- 130, First Quarter 2009.

[3] Z. Khan, J. Lehtomaki, K. Umebayashi and J. Vartiainen, "On the Selection of the Best Detection Performance Sensors for Cognitive Radio Networks", IEEE Signal Processing Letters, vol. 17, no. 4, pp. 359- 362, Apr. 2010.

[4] W. Zhang, R. K. Mallik and K. Ben Lataief, "Cooperative Shared Spectrum Sensing for Dynamic Cognitive Radio Networks", Proc. IEEE International Conference on Communications 2008 (ICC '08), Beijing, China, May. 19-23, 2008.

[5] J. Q. Song, Z. Y. Feng, D. Fan J. T. Xue and Z. M. Liu, "Optimal Parameters for Cooperative Spectrum Sensing in Cognitive Radio System", Proc. IEEE Wireless Communications and Networking Conference 2010 (WCNC '10), Sydney, Australia, Apr. 18-21, 2010.

[6] Q. Zhao, L. Tong, A. Swami, and Y. C. Chen, "Decentralized cognitive MAC for opportunistic spectrum access in Ad Hoc networks: A POMDP framework, IEEE J. on Selected Areas in Comm., vol. 25, no. 3, pp. 589C600, Apr. 2007.

[7] C. H. Lee and W. Wolf, "Multiple access-inspired cooperative spectrum sensing for cognitive radio", IEEE Military Communications Conference, Orlando, FL, October 2007.

[8] A. R. Biswas, T. C. Aysal, S. Kandeepan, D. Kliazovich and R. Piesiewicz, "Cooperative Shared Spectrum Sensing for Dynamic Cognitive Radio Networks", Proc. IEEE International Conference on Communications 2009 (ICC '09), Dresdon, Germany, Jun. 14-18, 2009.

[9] F. F. Digham, M.-S. Alouini and M. K. Simon, "On the Energy Detection of Unknown Signals over Fading Channels", Proc. IEEE Internation Conference on Communications 2003 (ICC '03), Anchorage, USA, May. 11-15, 2003, pp. 3575-3579

[10] Z. L. Wang, Z. Y. Feng, J. Q. Song, Y. Hu and P. Zhang, "A Practical Semi Range-based Localization Algorithm for Cognitive Radio", Proc. IEEE 71th Vehicular Technology Conference (VTC 2010-Spring), Taipei, China, May. 16-19, 2010.

[11] H. W. Kuhn, "The Hungarian Method for the Assignment and Transportation Problems", Naval Research Logistics Quarterly 2, 1955, pp 83-97.

[12] J. Munkres, "Algorithms for the Assignment and Transportation Problems", Journal of the Society for Industrial and Applied Mathematics, vol. 5 No. 1, Mar. 1957, pp. 32-38.

[13] R. E. Burkard and E. Cela, "Linear Assignment Problems and Extensions", Kluwer Academic Publishers, 1999. 